\title{
Design and Fabrication of Double Corrugated Waveguide for a Ka-band Traveling Wave Tube
}

\author{
Claudio Paoloni \\ Engineering Department, \\ Lancaster, UK, LA1 4YW
}

\author{
Mauro Mineo \\ E2v Technologies \\ Chelmsford, UK
}

\author{
Luke Bushnell \\ Mat Beardsley \\ Kai Parow-Souchon \\ Manju Henry \\ Peter G. Huggard \\ MMT Group, STFC Rutherford \\ Appleton Laboratory, Didcot, \\ OX11 0QX, United Kingdom
}

\begin{abstract}
The double corrugated waveguide is demonstrated to be a slow wave structure with easy fabrication and assembly. The design and fabrication of a Ka-band prototype is presented. The ability of the double corrugated waveguide to be bent through $90^{\circ}$ has permitted to build a compact slow wave structure with very good electrical behavior which is suitable for millimeter wave travelling wave tubes.
\end{abstract}

Keywords: traveling wave tube, double corrugated waveguide, sws, TWT, Ka-band.

\section{Introduction}

The increasing number of applications of traveling wave tubes (TWTs) at millimeter wave frequencies poses the challenge of balancing cost and performance [1 -2]. The diameter of helices for frequencies above $20 \mathrm{GHz}$, in the range of hundreds of $\mu \mathrm{m}$, makes assembly very challenging. Helix based TWTs have been demonstrated up to $70 \mathrm{GHz}$, but the volume production of TWTs in this frequency range is hindered by the high cost and low yield. The use of more easily assembled interaction structures is one approach to produce affordable TWTs.

The double corrugated waveguide (DCW) [3-5] is an all metal slow wave structure based on two parallel rows of pillars enclosed in a rectangular waveguide. The easy fabrication, straightforward assembly and robustness make this a promising millimeter wave helix replacement. This paper describes the design and fabrication of the DCW for a Ka- Band TWT [6].

\section{Design}

A DCW has been designed to operate in the frequency band $32-37 \mathrm{GHz}$. The dispersion curve was optimised to support an electron beam voltage of $12 \mathrm{kV}$ (Fig. 1). This beam voltage was chosen to limit the cost and the volume of the power supply.

The full interaction structure was designed to be easily fabricated and measured. The DCW needs a specific

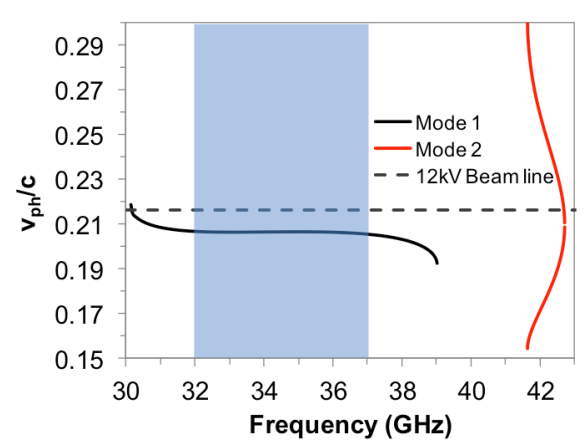

Fig. 1: Dispersion curve and beam line

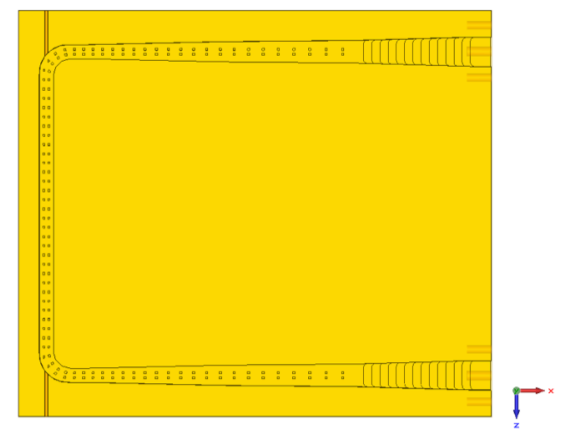

Fig. 2: Schematic of the DCW interaction structure coupling to transform the hybrid mode propagating into a $\mathrm{TE}_{10}$ mode at the rectangular waveguide flanges. The most effective coupler was found to be based on the tapering in height of a series of pillars at the end of the waveguide. However, the electron beam interaction is almost negligible in the tapered region, which for a linear device adds length to the magnetic confinement system and reduces the overall efficiency. The property of the DCW to be bent through $90^{\circ}$ allows placing the transition regions perpendicular to the gain section of the TWT. The TWT length is thus determined only by the active region, minimizing the dimensions of the focusing magnetic system. 


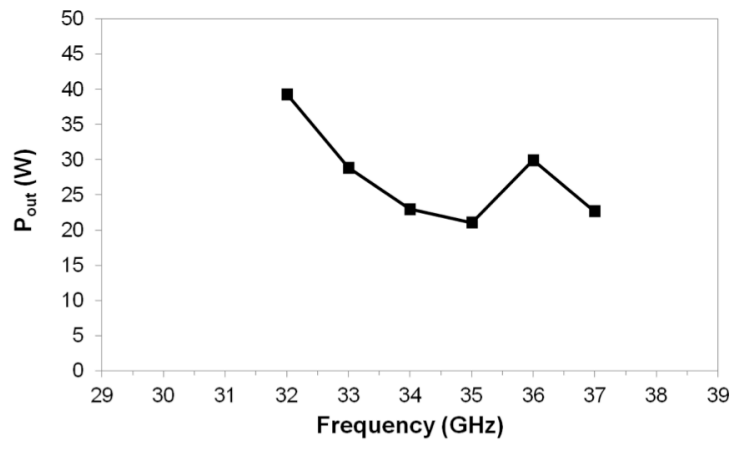

Fig. 3: Particle in cell simulation of the output power for 70 period DCW SWS

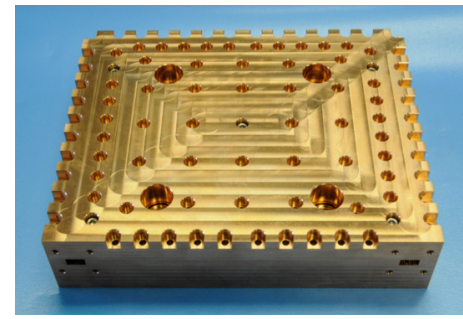

Fig. 4: Manufactured and assembled copper DCW

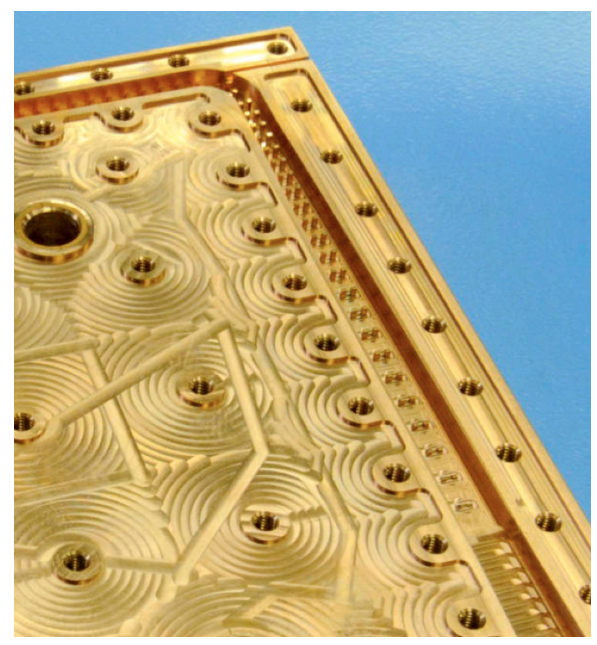

Fig. 5: Detail of the bend, the tapered coupler and the waveguide transition to the flange

A schematic of the interaction and coupling structures for such a DCW TWT is shown in Fig. 2. The pillars in the corners are shaped and placed to avoid the electron beam. The design of the DCW in the schematic was derived from MAGIC3D using particle in cell simulations. The number of periods was set as 70 . A very good output power, $>20 \mathrm{~W}$, was obtained with a beam current of $200 \mathrm{~mA}$. A magnetic focusing field of $0.3 \mathrm{~T}$ was applied to assure the proper beam confinement.

\section{Fabrication}

The DCW shown in Fig. 2 was fabricated, using a KERN Pyramid Nano precision CNC milling machine, from tellurium-copper alloy. Fig. 4 shows a photograph of the DCW assembled for measurement. Fig. 5 provides detail of the bend, the coupler and the transition to the flange. The precision $\mathrm{CNC}$ machining allows excellent definition of the designed structure, and VNA measurements confirmed a $\mathrm{S}_{11}$ better that $-20 \mathrm{~dB}$ in the operating frequency band.

\section{Conclusions}

The DCW has been demonstrated to be an effective slow wave structure which overcome the difficult fabrication and high cost of helical devices at millimeter wave frequencies. A novel approach to realizing a compact TWT with improved performance, by bending the DCW, is presented. This promising device demonstrates the potential importance of the DCW in the field of vacuum electron devices as a solution for a new family of affordable millimeter wave TWTs.

\section{Acknowledgements}

The authors are grateful to Andy Wood, Mike Duffield, Darrin Bowler and Paul John of e2v for their continued support and advice. The authors wish to acknowledge the funding received from STFC Grant ST/L003406/1. ATK's Alliance Partnership Program, which was provided via the use of their MAGIC software.

\section{References}

1. C. K. Chong and W. L. Menninger, "Latest Advancements in High-Power Millimeter-Wave Helix TWTs," IEEE Trans. on Plasma Science, vol.38, no.6, pp.1227,1238, June 2010

2. C. K. Chong et al., "Development of high-power Kaband and Q-band helix-TWTs," IEEE Trans. on Electron Devices, vol.52, no.5, pp.653,659, May 2005

3. M. Mineo and C. Paoloni, "Double-Corrugated Rectangular Waveguide Slow-Wave Structure for Terahertz Vacuum Devices," IEEE Trans. on Electron Devices, vol.57, no.11, pp. 3169-3175.

4. C. Paoloni et al., "Design and Realization Aspects of 1-THz Cascade Backward Wave Amplifier Based on Double Corrugated Waveguide," IEEE Trans. on Electron Devices, vol.60, pp.1236,1243, March 2013

5. C. Paoloni and M. Mineo, "Double Corrugated Waveguide for G-Band Traveling Wave Tubes,", IEEE Trans. on Electron Devices, vol.61, no.12, pp.4259,4263, Dec. 2014

6. C. Paoloni, M. Mineo, M. Henry, P. G. Huggard, "Double Corrugated Waveguide for Ka-Band Traveling Wave Tube," , IEEE Trans. Electron Devices, vol. 62, no.11, pp. 3851-3856, Nov. 2015. 